\title{
Sampled Data Robust Control of an Under-Actuated Electromechanical System with Input Delay
}

\author{
Hafsa Shafiq ${ }^{1, a}$ and Fahad M. Malik ${ }^{2}$ \\ ${ }^{1,2}$ National University of Sciences and Technology, Department of Electrical Engineering, 44000 Islamabad, Pakistan
}

\begin{abstract}
This paper focuses on sampled data control of an under-actuated electromechanical system with input delay, subjected to both parametric uncertainty and exogenous disturbances. The system is stabilized by a robust $\boldsymbol{H}_{\infty}$ controller that stabilizes the closed loop system and attains a specified level of perturbation attenuation for all permissible parametric uncertainty. The proposed $\boldsymbol{H}_{\infty}$ controller is designed for the orientation control of an underactuated electromechanical system which is a customized drill machine. The controller is designed on the basis of discrete time equivalent model of the system which is fully actuated and time invariant. The controller performance is evaluated on the basis of Monte Carlo simulations.
\end{abstract}

\section{Introduction}

The performance of a controller in a closed loop configuration is measured in terms of norm of the closed loop system. The synthesis of control system proceeds by finding a controller that optimizes the closed loop norm of the system. The development of $H_{\infty}, \mathrm{H}_{2}$ or LQG controllers have proven the usefulness and effectiveness of this approach. $H_{\infty}$ controller provides stabilization to a system with guaranteed robustness to disturbances while minimizing the $H_{\infty}$ norm of the closed-loop transfer matrix from the exogenous input to the output for a given plant G. This development was first introduced in control theory by the influential work of George Zames in 1981 [1]. Since then considerable advancements have been made in the field. See for example the work of Zames and Francis in 1983 [2], Glover and Doyle in 1988 [3] and Doyle, Glover, Khargonekar and Francis in 1989 [4].

The subject of preserving stability of a precisely stable system exposed to uncertainties or perturbations is thought to be a significant topic for study of the researchers. Considerable amount of literature has been presented concerning the issue of the stability of perturbed continuous and discrete time systems [5]-[7]. Lately the concern is more towards sampled data systems because of its widespread use of technology these days.

A sampled data control system involves a continuous time system being controlled by a discrete time controller with the involvement of $\mathrm{A} / \mathrm{D}$ and $\mathrm{D} / \mathrm{A}$ converters.

In this paper, sampled data control of an underactuated system with input delay is considered in the framework of $H_{\infty}$ control. The under-actuated system is a drill machine with customized actuation wherein two degrees of freedom are controlled using a single electromagnetic actuator. The system is also energized by perturbations of various types. The problem comprises of determining a sampled data robust controller that can minimize the $H_{\infty}$ norm of the closed loop system.

The customized actuation of the drill machine results in time varying actuation characteristics. The dill machine is completely actuated for a pulse of short duration for a fixed interval of time known as the actuation cycle, for the preferred orientation control during an entire revolution of the drill bit while it remains unactuated during the remaining interval. The discrete time robust controller is applied for the orientation control of the under-actuated drill machine. The controller determines the magnitude and phase (starting point) of the actuation pulse during the actuation cycle while maintaining the stability of the system in the presence of perturbations. The magnitude of the actuation pulse is determined using the $H_{\infty}$ controller design based on the discrete time equivalent model of the continuous time system which is fully actuated and time invariant [8]-[9] while the phase of actuation pulse additionally requires model based predicted states for its calculation.

The under-actuated drill machine system under observation is formerly controlled using the pole placement technique [10]. The novel $H_{\infty}$ control scheme presented in this paper will address the subject of input delay and disturbance rejection in addition to the stabilization of the drill bit at the desired reference. The controller thus designed is optimally robust for the system parameters and any external source of perturbation and uncertainties.

The paper is arranged in six sections starting with the introduction. Section 0 describes the continuous time dynamic model of the under-actuated system i.e. the drill machine accompanied with its under-actuation mechanism. Discrete time equivalent model of the system

\footnotetext{
ahafsashafiq@gmail.com
} 
with input delay is developed in Section 0. Section 0 consists of the robust control design, theory of $H_{\infty}$ optimization control and method to solve the Discrete Time Algebraic Riccati Equation. Perturbation analysis and various perturbation types are also discussed in Section 0. The simulation results for the orientation control of the under-actuated drill machine and thus the stabilization of the drill bit are illustrated in Section 0, which is followed by the conclusion in Section 0 and then references.

\section{System model}

The dynamic mathematical model of the drill bit is established taking into consideration its similarity with the spinning gyroscope.

\subsection{System dynamics}

The dynamic behaviour of the drill bit as that of a gyroscope is given by (1)

$$
\begin{aligned}
& J \ddot{\theta}_{x}+b \dot{\theta}_{x}+H \dot{\theta}_{y}=K_{i} \tau_{x} \\
& J \ddot{\theta}_{y}+b \dot{\theta}_{y}+H \dot{\theta}_{x}=K_{i} \tau_{y}
\end{aligned}
$$

where $\theta_{x}$ and $\theta_{y}$ represent the angular positions of the drill bit in radians about $x$ and $y$ axes respectively and $\tau_{x}$ and $\tau_{y}$ are the torques applied about these axes respectively, $J$ represents the moment of inertia, $b$ is the coefficient of friction, $H$ is the angular momentum defined by the expression $H=\left(J_{z}-J\right) \omega$, (where $\omega$ is the spin frequency and $J_{Z}$ is the moment of inertia of the drill bit about $z$ axis) and $K_{i}$ is the torque constant.

To write the system in conventional state space notation, define the angular positions $\theta_{x}$ and $\theta_{y}$ to be the state variables $x_{1}$ and $x_{3}$ and the derivatives of the angular positions to be $x_{2}$ and $x_{4}$ respectively. The applied torque about $x$ and $y$ axes i.e. $\tau_{x}$ and $\tau_{y}$ are considered as input components $u_{1}$ and $u_{2}$ respectively.

$$
\begin{aligned}
& \dot{x}_{1}=x_{2} \\
& \dot{x}_{2}=-\frac{b}{J} x_{2}-\frac{H}{J} x_{4}+\frac{K_{i}}{J} u_{1} \\
& \dot{x}_{3}=x_{4} \\
& \dot{x}_{4}=-\frac{b}{J} x_{4}+\frac{H}{J} x_{2}+\frac{K_{i}}{J} u_{2}
\end{aligned}
$$

The output of the system are the angular positions $x_{1}$ and $x_{3}$, that need to be stable at the reference $r$. The summarized matrix representation of the system (2) is

$$
\begin{aligned}
& \dot{x}=A x+B(t) u \\
& y=C x
\end{aligned}
$$

where

$$
A=\left[\begin{array}{cccc}
0 & 1 & 0 & 0 \\
0 & -b / J & 0 & -H / J \\
0 & 0 & 0 & 1 \\
0 & H / J & 0 & -b / J
\end{array}\right]
$$

$$
B=\left[\begin{array}{cc}
0 & 0 \\
K_{i} / J & 0 \\
0 & 0 \\
0 & K_{i} / J
\end{array}\right] \text { and } C=\left[\begin{array}{cccc}
1 & 0 & 0 & 0 \\
0 & 0 & 1 & 0
\end{array}\right]
$$

The matrix $B(t)$ is a time varying input matrix, due to the under-actuation characteristics of the system, with magnitude $\mathrm{B}$.

\subsection{Actuation mechanism}

The drill machine under discussion has customized construction and enable the use of individual pair of electromagnetic poles for controlling the two degrees of freedom resulting in an under-actuated system. The detailed construction and operation of the drill machine is explained in [10]. The rotor of the drill machine is a ring shaped permanent magnet and its stator has a single coil wound over it. Excitation of the stator coil produces orientation control field whose interaction with rotor field is used to produces torque, thus causing precession of the bit because of its gyroscopic action. This results in changing the orientation of the drill bit.

The stator electromagnet is energized when the permanent rotor magnet is aligned with the precession axis (field of the permanent rotor magnet is perpendicular to the desired projection vector). This time interval $L$ marks the center of actuation pulse and is a fraction of the time period $T=2 \pi / \omega$ of an entire revolution of drill bit. The input magnitude stays zero order held throughout this portion of the time period. The actuation pulse is provided to the system with an actuation delay $\Delta$ that is dependent on the desired reference $r$. The system is kept unactuated for remaining interval $T-L$ in the actuation cycle given in Figure 1.

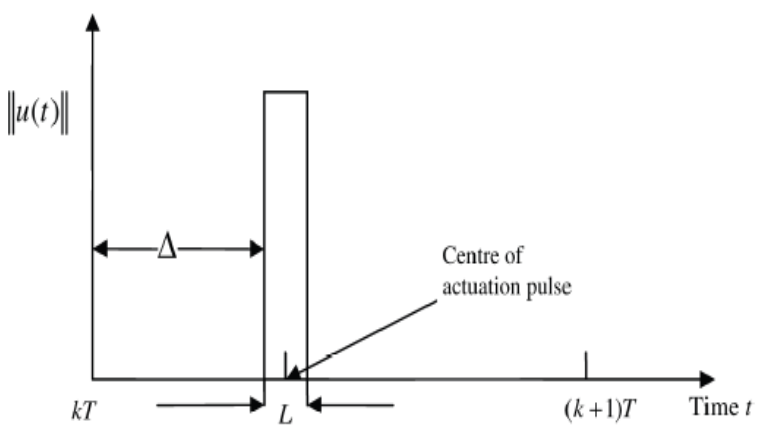

Figure 1. A Single Actuation Cycle

\subsection{Delay factor}

Time delays are habitually encountered in practical control systems. In the previous several years, ample amount of consideration is paid to the knowledge of stabilization of perturbed systems having delays in control input [11]-[12]. The input delay in a system can be due to the delays in the transmission from sensor towards controller or controller towards actuator etc. They cause dynamics of the system to respond to the applied input after certain interval of time and hence, limitation in the response speed. If the under-actuated 
linear system has input delay of $N L$ seconds. It is assumed that $N L<T$. The continuous time model then becomes

$$
\begin{aligned}
& \dot{x}(t)=A x(t)+B(t) u(t-N L) \\
& y(t)=C x(t)
\end{aligned}
$$

\section{Discrete time equivalent model}

The discrete time equivalent model of the drill machine system (5) is established by considering a complete actuation cycle $[k T+\Delta, k T+\Delta+T]$ as one discrete step. The under-actuated drill machine under discussion is also assumed to have input delay. Thus, a transformation presented in literature in recent past [13] is modified for the class of under-actuated systems with input delay that results in an equivalent model without input delay.

The discrete time equivalent model for the continuous time system of the drill machine without input delay is already discussed in [10], therefore, only the transformation for the delayed input part is presented here. For developing the equivalent model of (5), one actuation cycle is divided into two periods. In first phase, the time duration $t \in[k T+\Delta, k T+\Delta+L]$ is taken, wherein the system is actuated using the short duration pulse of constant magnitude B. For the second phase, the interval $t \in[k T+\Delta+L, k T+\Delta+T]$ is used, wherein the system remains unactuated.

The system state at $t=k T+\Delta+L$ in terms of its state at $t=k T+\Delta$ is

$$
\begin{aligned}
x(k T+\Delta+L)= & A_{d 1} x(k T+\Delta)+ \\
& B_{d 1} u(k T+\Delta-N L)
\end{aligned}
$$

Representing time $k T+\Delta$ by $i, k T+\Delta+L$ by $i+$ 1 and switching to conventional bracket notation for discrete time representation, (6) is written as

$$
x[i+1]=A_{d 1} x[i]+B_{d 1} u[i-N]
$$

where

$$
A_{d 1}=\exp (A L) \text { and } B_{d 1=} B \int_{0}^{L} \exp (A(L-\sigma)) d \sigma \text {. }
$$

The discrete time system (7) is transformed into an equivalent system without input delay by the following change of variables

$$
z[i]=x[i]+\sum_{j=i-N}^{i-1} A_{d 1}^{-(j-i+N+1)} B_{d 1} u[j]
$$
by

Dynamics of the transformed system are represented

$$
z[i+1]=x[i+1]+\sum_{j=i-N}^{i-1} A_{d 1}^{-(j-i+N+1)} B_{d 1} u[j+1]
$$
by

The transformed system without input delay is given

$$
z[i+1]=A_{d 1} z[i]+A_{d 1}^{-N} B_{d 1} u[i]
$$

Likewise considering the second interval, the state of the system at $t=k T+\Delta+T$ in terms of the state at $t=k T+\Delta+L$ is

$$
x(k T+\Delta+T)=A_{d 2} x(k T+\Delta+L)
$$

where $A_{d 2}=\exp (A(T-L))$.

In this interval, the system is unactuated, thus $u=0$. Let $i+M=k T+\Delta+T$, then

$$
z[i+M]=A_{d 1}^{M-1} z[i+1]
$$

Since $A_{d 1}=\exp (A L)$, then $A_{d 1}^{M-1}=\exp (A(T-L)$.

The discrete time equivalent model for the drill machine (5) considering the complete actuation cycle $[k T+\Delta, k T+\Delta+T]$ as a unique discrete step and without input delay is

$$
\begin{aligned}
z[m+1] & =A_{d 2} A_{d 1} z[m]+A_{d 2} A_{d 1}^{N} B_{d 1} u[m] \\
& =A_{d}^{\prime} z[m]+B_{d}^{\prime} u[m]
\end{aligned}
$$

where $A_{d}^{\prime}=A_{d 2} A_{d 1}$ and $B_{d}^{\prime}=A_{d 2} A_{d 1}^{-N} B_{d 1}, m=k T+$ $\Delta$ and $m+1=k T+\Delta+T$.

\section{Robust control design}

\section{1 $\mathrm{H}_{\infty}$ control}

$H_{\infty}$ optimization problem is formulated as the task of formulating a stabilizing controller $K$, that minimizes the $H_{\infty}$ norm of the closed-loop transfer matrix from the exogenous input $\omega$ to the output $z$ for a given plant $G$. Figure 2 shows a standard control system.

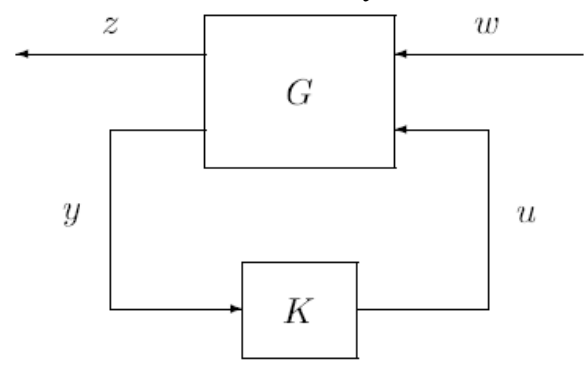

Figure 2. Standard Control System Configuration

This system is described as

$$
\begin{aligned}
{\left[\begin{array}{l}
z \\
y
\end{array}\right]=G(s)\left[\begin{array}{l}
\omega \\
u
\end{array}\right] } & =\left[\begin{array}{ll}
G_{11}(s) & G_{12}(s) \\
G_{21}(s) & G_{22}(s)
\end{array}\right]\left[\begin{array}{l}
\omega \\
u
\end{array}\right] \\
u & =K(s) y
\end{aligned}
$$

The input $\omega$ is an exogenous input indicating the disturbance effect in the system. The output $z$ is the control system output, whose dependency on $\omega$ needs to be optimized. The output $y$ is a measurement of the system that helps in selecting the control input $u$, which consecutively is the way to reduce the influence of $\omega$ on $z$ [14].

The closed loop transfer function from $\omega$ to $z$ is specified by the linear fractional transformation.

$$
z=F_{l}(G, K) \omega
$$

where

$$
F_{l}(G, K)=G_{11}+G_{12} K\left(I-G_{22}\right)^{-1} G_{21}
$$


$H_{\infty}$ control involves the minimization of the $H_{\infty}$ norm of the LFT (16). It is one of the popular performance index in optimal control theory given as

$$
\left\|F_{l}(G, K)\right\|_{\infty}=\max _{\omega} \bar{\sigma}\left(F_{l}(G, K)(j \omega)\right)
$$

Let $\gamma_{\min }$ be the minimum value of $F_{l}(G, K)$, it is usually preferred to design an $H_{\infty}$ suboptimal control problem i.e. given a $\gamma>\gamma_{\min }$, the stabilizing controller $K$ is found such that

$$
\left\|F_{l}(G, K)\right\|_{\infty}<\gamma
$$

\subsection{Discrete time Algebraic Riccati Equation}

Let $(A, B)$ is stabilizable, then the internally stabilizing controller $K$ that satisfies (18) is obtained, [15] iff the following generalized discrete time Algebraic Riccati Equation

$$
X_{\infty}=Q+A_{d}^{\prime} X_{\infty} A_{d}-L^{\prime} M^{-1} L
$$

with

$$
\begin{aligned}
& J=\left[\begin{array}{ll}
I & 0 \\
0 & -\gamma^{2} I
\end{array}\right] \\
& L=D_{d}^{\prime} J C_{d}+B_{d}^{\prime} X_{\infty} A_{d} \\
& M=D_{d}^{\prime} J D_{d}+B_{d}^{\prime} X_{\infty} B_{d} \\
& Q=C_{d}^{\prime} J C_{d} \\
& R=D_{d}^{\prime} J D_{d}
\end{aligned}
$$

has a solution such that

$$
\begin{aligned}
& A_{d}-B_{d} M^{-1} L \quad \text { is asmptotically stable } \\
& X_{\infty} \geq 0 \\
& M_{1}-M_{2}^{\prime} M_{3}^{-1} M_{2}=\nabla<0
\end{aligned}
$$

The $M$ and $L$ matrix are partitioned as

$$
M=\left[\begin{array}{ll}
M_{1} & M_{2}^{\prime} \\
M_{2} & M_{3}
\end{array}\right], \quad L=\left[\begin{array}{l}
L_{1} \\
L_{2}
\end{array}\right]
$$

If the assumptions (20) are satisfied, the gain of the controller $K$ is given as

$$
K=\left(R+B_{d}^{\prime} X_{\infty} B_{d}\right)^{-1} B_{d}^{\prime} X_{\infty} A_{d}
$$

\subsection{Control input}

The control law for the stabilization of the drill bit is developed based upon discrete time equivalent model of the system without input delay (13). The optimal feedback gain matrix $K$ is calculated using the robust $H_{\infty}$ controller. The feedback control input is calculated in terms of the original coordinates

$$
\begin{aligned}
u[m] & =-K z[m]+\operatorname{Pr} \\
& =-K\left(x[i]+A_{d 1}^{-N} B_{d 1} u[i-N]\right)+\operatorname{Pr}
\end{aligned}
$$

where $P$, the reference pre-scalar [16], and the term $P r$ is used to stabilize the system at the preferred reference position rather than the origin. It is given as

$$
P=\left[C_{d}\left(I-A_{d}+B_{d} K\right)^{-1} B_{d}\right]^{-1}
$$

The delayed input for the system is

$$
\begin{gathered}
u[i-N]=-K\left(x[i-N]-A_{d 1}^{-N} B_{d 1} u[i-N-1]+\operatorname{Pr}\right. \\
\text { Since } u[i-N-1]=0 \\
u[i-N]=-K x[i-N]+\operatorname{Pr}
\end{gathered}
$$

The actuation pulse magnitude is constant and is equal to $\mathrm{B}$ as described in Section 0 . The centre of actuation is the instant when the permanent rotor magnet is aligned with the precession axis. At this point, the rotor permanent magnet is perpendicular with the control input phase i.e. the torque vector. The centre of actuation pulse is marked as the discrete index $i$, where the following equation is fulfilled.

$$
\alpha[i]=\tan ^{-1}\left(\frac{u_{2}[i]}{u_{1}[i]}\right)+\frac{\pi}{2}
$$

where $u_{p}[i]$ is the $p^{t h}$ component of the vector $u[i]$.

The discrete-time equivalent model equation is derived for an interval of $T$ seconds (specified by the discrete index $m$ ) that characterizes a complete revolution of the drill bit. However, the sampling time of states is $L$ seconds (specified by discrete index $i$ ) that represents a fraction of the complete revolution time $T$. Consequently, for the system model and the controller, there are separate discrete-time measures.

The condition (26) symbolizes the centre of actuation however, in practice, the actuation needs to start $L / 2$ seconds earlier than centre of actuation. This results in the actuation of the system as non-causal, so model-based predicted state approximations at the following index is used to overcome the non-causality.

$$
\hat{x}[i+1]=A_{d 1} x[i]+B_{d 1} u[i]
$$

The controller then use this state estimate at the next sample $\hat{x}[i+1]$ in determining whether the point $t=$ $i L+L / 2$ would be the point of actuation or not. Mathematically

$$
\text { If }\left|\hat{\alpha}[i+1]-\left[\tan ^{-1}\left(\frac{\hat{u}_{2}[i+1]}{\hat{u}_{1}[i+1]}\right)+\frac{\pi}{2}\right]\right|<\frac{\omega L}{2}
$$

$u(t)=-K \hat{x}\left(i L+\frac{L}{2}\right)+G r \quad i L+\frac{L}{2} \leq t<(i+1) L+\frac{L}{2}$

else

$$
u(t)=0
$$

where the centre of actuation for the next sample is $\hat{\alpha}[i+1]=\alpha[i]+\omega(L / 2) \quad$ and $\quad \hat{x}(i L+L / 2)=$ $\exp (A(L / 2)) x(i L)$. The tolerance $\omega L / 2$ in calculating the estimated input phase is presented to overcome any imprecisions of state prediction.

\subsection{Perturbation analysis}

Uncertainties are the inherent component of a real control system, classified primarily as disturbance signals and dynamic perturbations. The former comprises of input and output exogenous disturbances. Input disturbances are mostly due to the sensor noise while output disturbances are caused by the measurement noise. Let $\omega$ and $v$ represent the input and output disturbances respectively, then the model of the system is given in (30) 


$$
\begin{aligned}
& \dot{x}=A x+B u+W \omega \\
& y=C x+D u+V v
\end{aligned}
$$

Dynamic perturbations generally represent the inaccuracies between the actual dynamics of the system and its mathematical model. They are further classified as unstructured uncertainties (include un-modelled, highfrequency or neglected system dynamics), parametric uncertainties (inaccurate description of component characteristics), structured uncertainty (combined scenario of the above two with the uncertain block of diagonal form) [17]. Let $\Delta$ represents the perturbation matrix, $G_{o}$ is the nominal system and $G_{p}$ is the perturbed system, various unstructured uncertainties are listed in Table 1 .

Table 1. Various Unstructured Perturbation

\begin{tabular}{|c|c|}
\hline Perturbation & Formula \\
\hline Additive & $G_{p}(s)=G_{o}(s)+\Delta(s)$ \\
\hline Input multiplicative & $G_{p}(s)=G_{o}(s)[I+\Delta(s)]$ \\
\hline Output Multiplicative & $G_{p}(s)=[I+\Delta(s)] G_{o}(s)$ \\
\hline $\begin{array}{c}\text { Inverse additive } \\
\text { Inverse input } \\
\text { multiplicative }\end{array}$ & $\left.\left(G_{p}(s)\right)^{-1}=\left(G_{o}(s)\right)^{-1}+\Delta(s)\right)^{-1}=[I+\Delta(s)]\left(G_{o}(s)\right)^{-1}$ \\
\hline $\begin{array}{c}\text { Inverse output } \\
\text { multiplicative }\end{array}$ & $\left(G_{p}(s)\right)^{-1}=\left(G_{o}(s)\right)^{-1}[I+\Delta(s)]$ \\
\hline
\end{tabular}

\section{Simulation results}

The various parameters of the under-actuated system of the drill machine in (2) used for simulation are: $J=$ $4 \mathrm{Kgm}^{2}, J_{z}=5 \mathrm{Kgm}^{2}, \omega=400 \pi \mathrm{rad} / \mathrm{sec}, K_{i}=1$ and $b=100$. The drill machine is initially at rest and desired reference for stabilization is selected to be $r=[0.4,0.3]^{T}$. The time period of a single revolution is $T=2 \pi / \omega$ sec while the duration of actuation and the sampling interval is $L=2 \pi / 16 \omega \mathrm{sec}$. Applying the discretization technique explained in Section 0, the state space matrices of the system (13) are

$$
\begin{aligned}
A_{d}^{\prime} & =\left[\begin{array}{cccc}
1 & 0.0036 & 0 & -0.0014 \\
0 & 0.4289 & 0 & -0.4289 \\
0 & 0.0014 & 1 & 0.0036 \\
0 & 0.4289 & 0 & 0.4289
\end{array}\right], \\
B_{d}^{\prime} & =\left[\begin{array}{cc}
0.0026 & -0.0009 \\
0.3764 & -0.3246 \\
0.0009 & 0.0026 \\
0.3246 & 0.3764
\end{array}\right] \times 10^{-4}
\end{aligned}
$$

For examining the robustness of the suggested control technique, the resulting system is perturbed with different types of disturbances as given in Section 0 and controller matrix $K$ is designed on the basis of $H_{\infty}$ optimization theory described in Section 0 . The system is simulated with $\pm 25 \%$ perturbations in the nominal value of its parameters and disturbances. For each analysis, 10,000 Monte Carlo Simulations are performed on the system, out of which maximum and minimum bounds on the angular positions are plotted and are presented in this section. The results show that for all the disturbances, the drill bit stabilizes at the desired reference, i.e. $r=$ $[0.40 .3]^{T}$ and there is no significant change in the nature of the response.

A sinusoidal signal is added as the input disturbance $\omega$ in the system (30) and the response is observed for various values of amplitude and frequency of the added disturbance signal. The system is also assumed to have $\pm 25 \%$ perturbations in its system matrix and also in the added disturbance signal.
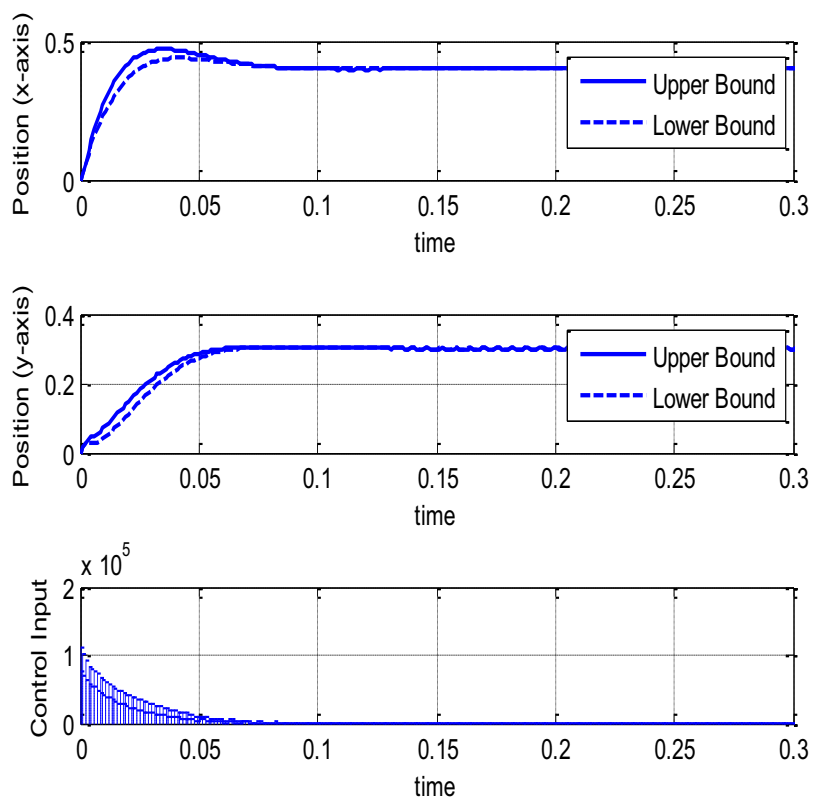

Figure 3. Stabilization of Drill Bit with Input Delay in the presence of Sinusoidal Input Disturbance with 25\% Perturbations

The results show that, increasing the peak to peak amplitude and frequency of the disturbance signal simultaneously, the desired reference is achieved in a fine behaviour as shown in Figure 3. However, at low frequency, $10 \mathrm{~Hz}$; increasing the peak to peak amplitude of input disturbance to 1000 , increases the magnitude of reference. Increasing the amplitude further, the response becomes sinusoidal. In this situation, the response oscillates with the desired reference as the mean position. Increasing the amplitude and frequency to very large values distort the response shape. The results vary in a similar manner when square input disturbance and sinusoidal or square output disturbances are added in the system (30) and their effects are observed by changing the amplitude and frequency of the disturbance signals.

Random signals are added as the input disturbance with $\pm 25 \%$ perturbations in (30) and their effect is observed on the system response as shown in Figure 4. 

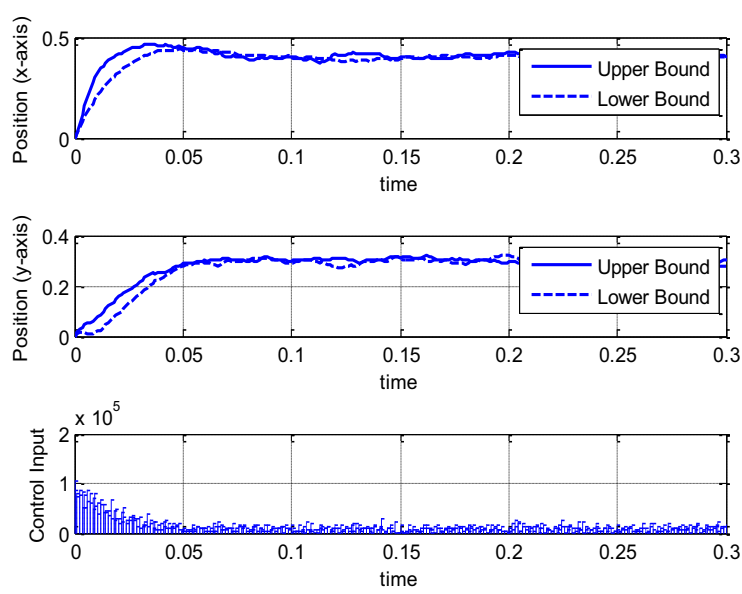

Figure 4. Stabilization of Drill Bit with Input Delay in the presence of Random Input Disturbance with 25\% Perturbations

The system response with additive perturbation is shown in Figure 5. The perturbation matrix $\Delta$ is considered to be 10 percent of the nominal value of the system matrix having $\pm 25 \%$ perturbations. The simulation shows that the system is stabilized at the desired reference.
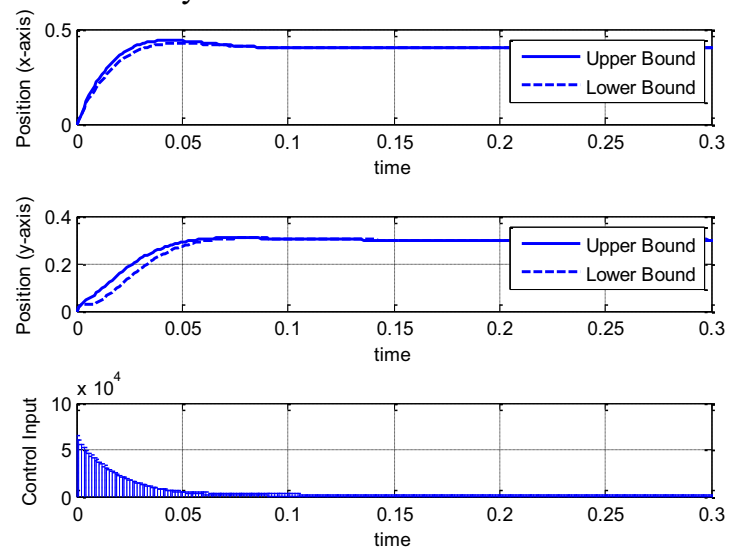

Figure 5. Stabilization of Drill Bit with Input Delay in the Presence of $25 \%$ Additive Perturbations

The system is simulated with output multiplicative perturbation having the perturbation matrix $\Delta$ to be 10 percent of the nominal value of the system matrix with $\pm 25 \%$ perturbations. Figure 6 shows that the system is stabilized at the desired reference with sufficient accuracy.
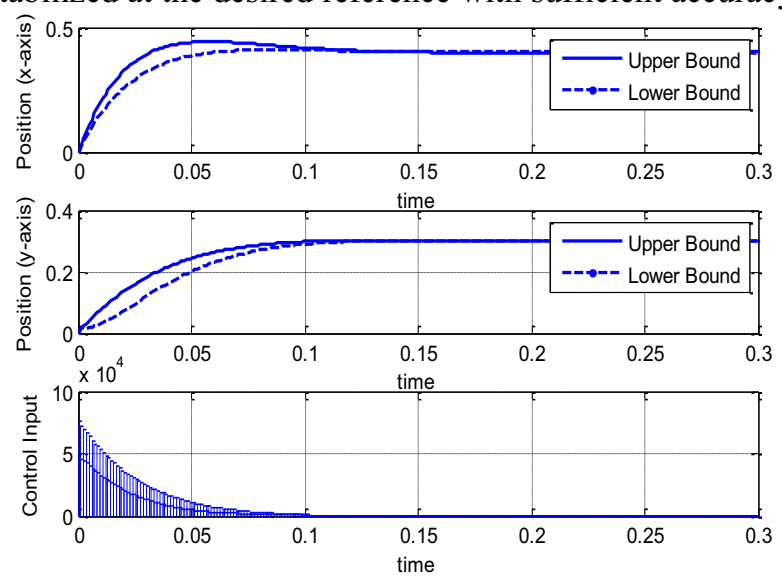

Figure 6. Stabilization of Drill Bit with Input Delay in the Presence of $25 \%$ Output Multiplicative Perturbations

\section{Conclusion}

$H_{\infty}$ optimization technique is used for the orientation control of the under-actuated drill machine with input delay and also accompanied with system perturbations. The simulation results clearly indicates that the system is stabilized at the desired reference with the help of $H_{\infty}$ controller. $H_{\infty}$ control synthesis is found to guarantee robustness and good performance in terms of sensitivity and provides high disturbance rejection. The advantage of $H_{\infty}$ control over state feedback is that it does not specify exactly where the closed loop eigenvalues should be, rather it specify some kind of performance objective function to be optimized. The work presented in the paper would interest the researchers in the future to enhance the system by adding a state observer that would incorporate the possibility of the system to work in case all the states are not available as direct measurement, which is generally the situation in a practical control system. A state observer would behave as a subsystem and performs an estimation of the state variables on the basis of the output measurement and the control variables. The observed state vector would then be used to generate the optimal control vector.

\section{References}

1. G. Zames, Feedback and Optimal Sensitivity: Model Reference Transformations, Multiplicative Seminorms, and Approximate Inverses. IEEE Trans. Autom. Control. 26(2), pp. 301 - 320, (1981, Apr.).

2. G. Zames, B. A. Francis, Feedback, minimax sensitivity, and optimal robustness. IEEE Trans. Autom. Control. 28(5), pp. 585 - 601. (1983, May).

3. K. Glover, J.C. Doyle, State-space formulae for all stabilizing controllers that satisfy an $\mathrm{H}_{\infty}$-norm bound and relations to risk sensitivity. Syst. \& Control Lett. (11), pp. 167-172, (1988).

4. J. C. Doyle, K. Glover, P. P. Khargonekar and B. A. Francis, State space solutions to standard $\mathrm{H}_{2}$ and $\mathrm{H}_{\infty}$ control problems. IEEE Trans. Autom. Control. 34(8), pp. $831-847$, (1989, Aug.).

5. I. K. Konstantopoulos, P. J. Antsaklis, Robust stability of linear continuous and discrete time systems under parametric uncertainty, Elect. Eng. Univ. Notre Dame, Tech. Rep. ISIS-94-006, (1994, Mar.).

6. S. R. Kolla, R. K. Yedavalli \& J. B. Farison, Robust stability bounds on time-varying perturbations for state-space models of linear discrete-time systems. Int. J. Control. 50(1), pp. 151 - 159, (2007, Apr.).

7. Q. L. Han, Robust Stability for a Class of Linear Systems with Time-Varying Delay and Nonlinear Perturbations. Comput. \& Math. Applicat. 47(8-9), pp. 1201 - 1209, (2004, Apr.-May).

8. D. S. Bernstein, C.V. Hollot, Robust stability for sampled-data control systems. Syst. \& Control Lett. 13(3), pp. 217 - 226, (1989, Sept.).

9. T. Chen, B. Francis, Optimal Sampled-Data Control Systems, 1st ed., Springer-Verlag, London, pp. 37 40, 1994. 
10. M. B. Malik, F. M. Malik, K. Munawar, Orientation control of a 3-D under-actuated drill machine based on discrete-time equivalent model. Int. J. Robotics \& Automat. 27(4).

DOI: 10.2316/Journal.206.2012.4.206-3324, (2012).

11. W. H. Kwon, A. E. Pearson, Feedback Stabilization of Linear Systems with Delayed Control. IEEE Trans. Autom. Control. 25(2), pp. 266 - 269, (1980, Apr.).

12. J. H. Kim, E. T. Jeung, H. B. Park, Robust control for parameter uncertain delay systems in state and control input. Automatica, 32(9), pp. 1337 - 1339, (1996, Sept.).

13. Y. Xia, G.P. Liu, P. Shi, J. Chen, D. Rees, J. Liang Sliding mode control of uncertain linear discrete time systems with input delay. Control Theory Applicat., IET, 1(4), pp. 1169 - 1175, (2007, July).

14. S. Skogestad, I. Postlethwaite, Multivariable Feedback Control: Analysis \& Design, 2nd ed., Wiley, Hoboken, New Jersey, pp. 371 - 392, 2005.

15. M. Green, D. J.N. Limebeer, Linear Robust Control, Pearson Education, Inc., New York, pp. 505 - 507, 1995.

16. Mechanical Engineers' Handbook: Instrumentation, Systems, Controls, and MEMS, Vol. 2, 3rd ed. John Wiley \& Sons, Inc., pp. 5, 2006.

17. D.-W. Gu, P. Hr. Petkov and M. M. Konstantinov, Robust Control Design with MATLAB, SpringerVerlag, London, pp. $24-27,2005$. 